\title{
LOCAL POLE FIGURES IN THE TEM- MEASUREMENT AND ODF DETERMINATION
}

\author{
HASSO WEILAND and S. PANCHANADEESWARAN \\ Alcoa Technical Center, Alcoa Center, PA, 15069, U.S.A.
}

Techniques for the measurement of local pole figures in the Transmission Electron Microscope (TEM) are summarized and applications to deformed aluminum are given. Local pole figures are obtained by measuring the intensity distribution along ring patterns in Selected Area Diffraction (SAD) separately for various tilts of the specimen with respect to the incident electron beam. The computer control of the TEM allows a data acquisition in reasonable time. The measured intensities are corrected either experimentally or analytically for the increase of sampled volume and for the increased absorption with increasing tilt angle of the specimen. The application of orientation distribution functions (ODF) using the series expansion technique in conjunction with the positivity method allows a quantitative texture analysis of the pole figures.

KEY WORDS Texture, pole figure, ODF, postitivity method, TEM, electron diffraction, SAD, aluminum.

\section{INTRODUCTION}

There are times when it is necessary to measure crystallographic texture on an extremely local scale. For example, heavily deformed or partially recrystallized materials show an inhomogeneous microstructure, where the texture varies with location in the sample. Therefore the measurement of the orientation distribution on a very local scale is necessary to understand, for example, the origin of preferred orientations during recrystallization or the formation of shear bands, transition bands, etc. In the single- and multilayered thin films used for electronic packaging, polycrystalline solar cells, and in coatings, X-ray diffraction based texture measurements are often not successful but satisfactory measurements can be obtained by electron diffraction.

Local or microscale texture measurements are mainly performed by electron diffraction techniques involving Scanning (SEM) or Transmission (TEM) Electron Microscopy. Advantages of the determination of orientations by electron microscopy are the high spatial resolution and the ability to image the microstructure of the sampled area.

In a TEM, information on the local texture of a material is obtained either by the measurement of individual grain orientations from Kikuchi patterns (Weiland, 1985) or by the measurement of local pole figures (Schwarzer, 1982; Humphreys, 1983; Schwarzer and Weiland, 1985). Local pole figures are obtained by the measurement of the intensity in selected area diffraction (SAD) patterns for different geometries of the sample with respect to the incident electron beam. 
In most cases a quantitative texture interpretation from a single pole figure is not possible. Therefore orientation distribution functions (ODF) have to be calculated from these data. While the ODF determination from a set of incomplete pole figures is routine in the case of X-ray and neutron diffraction techniques, this is difficult for pole figures obtained by electron diffraction. The difficulty lies in the necessary correction of the measured intensities with respect to the electron sample interaction. Also it is not possible to cover the complete pole sphere with the measurements, mainly because of mechanical constraints in the TEM gonoiometer. Additionally, the shape of this unmeasured area complicates the numerical calculations.

It is the aim of this paper, to review the basics of the measurement techniques and to outline the methods for a quantitative analysis of local pole figures thus obtained.

\section{MEASUREMENT TECHNIQUES \\ Selected Area Diffraction}

Pole figures in the TEM are obtained by the measurement of the diffracted intensity along the rings obtained by selected area diffraction (Figure 1). A SAD pattern is obtained from the illuminated sample region by selecting an area of interest in the sample with the help of an aperture in the intermediate image plane. Bragg diffraction then gives for each set of lattice planes $\{H K L\}$ a separate diffraction ring, with radius dependent on the Bragg angle. The measurement is repeated for the whole range of eucentric tilts of the TEM goniometer. A single-tilt specimen holder is necessary to retain eucentricity with all tilt angles. For certain applications, a rotation-tilt holder can be used.

The size of the specimen area for analysis is a function of the specimen thickness and therefore depends on the specimen preparation. The largest area technically possible is about 0.5 to $1 \mathrm{~mm}$ in diameter, which can be used for thin films. In samples prepared by electrothinning, the maximum area achieved so far is about $0.1 \mathrm{~mm}$ in diameter. The minimal area for a local pole figure analysis depends on the measurement technique and will be discussed later.

a)

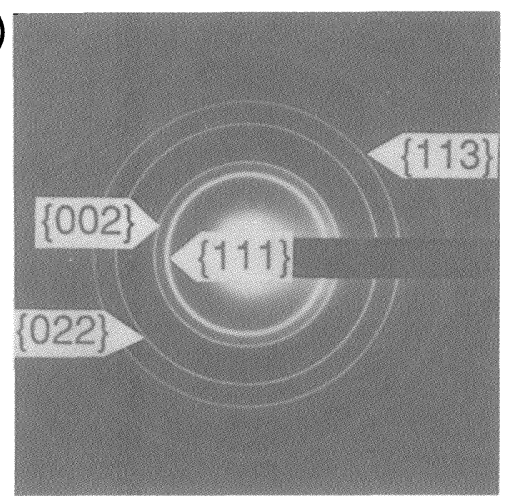

b)

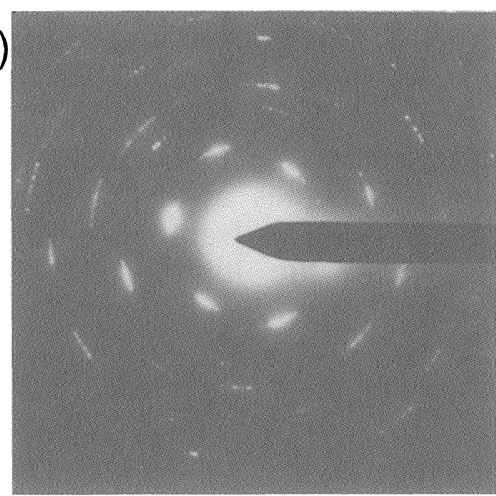

Figure 1 SAD diffraction pattern of a) evaporated aluminum (randomly texture) and b) of cold rolled aluminum (strongly textured). 


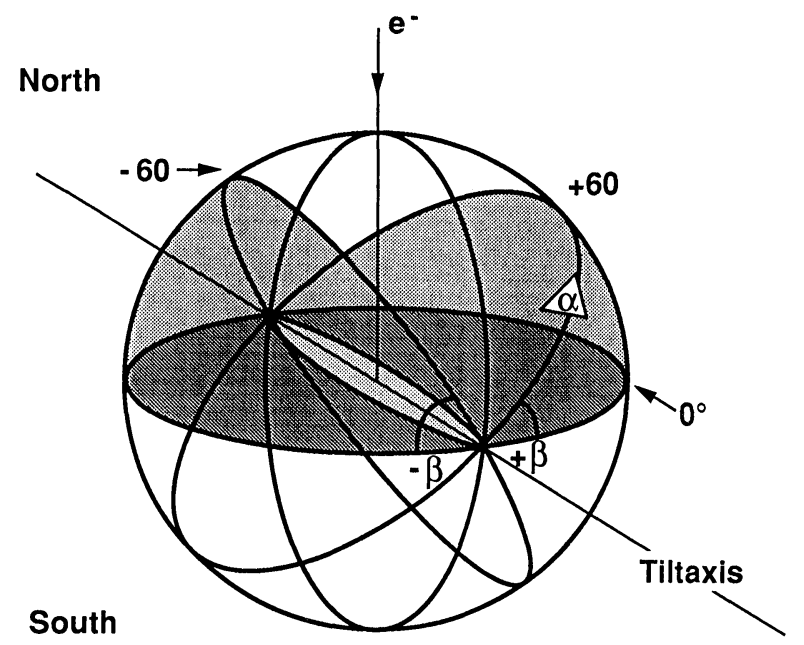

Figure 2 Pole sphere showing the areas detected by electron diffraction. $e^{-}$is the incident electron beam. The horizontal plane shows the stereographic projection of the entire pole sphere, with the lens-shaped unmeasured area (bright area). $\beta$ corresponds to the tilt angle of the TEM goniometer, +60 and -60 are the maximum tilt angles, $\alpha$ is the azimuth on each diffraction ring. North and South are the upper and the lower part of the pole sphere.

The intensity along a diffraction ring is, as a result of the small Bragg angles obtained by electron diffraction, virtually the pole density along a great circle on the pole sphere. Because of the limitations in the tilt range $\beta$ by the object space area in the TEM goniometer, the pole sphere can only be covered for certain values of $\beta$. The limited tilt range will leave a lens-shaped unmeasured area in the center of the stereographic projection. Most analytical TEMs allow an eucentric tilt in the range of $0^{\circ}$ to $\pm 60^{\circ}$ (Figure 2).

The intensity of each diffraction ring is a function of various parameters such as absorption and dynamical electron-specimen effects, but also depends on the texture of the analyzed sample area. Furthermore, this intensity distribution varies with different geometries of the sample with respect to the incident electron beam. This means that the intensity for a given azimuth $\alpha$ changes with different tilt angles $\beta$ of the goniometer. Besides the increasing diffracting volume with increasing tilt angle of the goniometer, the change in intensity depends on the real local texture. Therefore no conclusions on the texture of a material can be drawn from a single SAD pattern obtained at only one tilt angle of the TEM goniometer.

The concept of pole figure measurement in a TEM. The general concept of measuring intensity along diffraction rings in a TEM is based on the fact that electrons can be deflected by electromagnetic fields. Thus it is possible to deflect under computer control the diffraction pattern across a stationary detector in the TEM camera chamber, such that consecutive points on a great circle of the pole sphere are measured.

The deflected intensity is measured either by a Faraday cup or by the current from the fluorescent screen. The currents measured by a detector are in the 
picoampere range and have to be measured by a fast and sensitive current amplifier.

The two established techniques for the measurement of local pole figures are the post- and pre-specimen deflection techniques.

In the post-specimen deflection technique, the entire diffraction pattern is deflected with the help of deflection coils below the objective lens (Figure 3). These are the diffraction spot alignment coils conventionally provided on a microscope but modified to give an enlarged displacement capability. The intensity is measured with a Faraday cup. With this technique, the angular resolution can be adjusted by changing the camera length. An angular resolution of $2^{\circ}$ to $5^{\circ}$, needed for standard ODF analysis, can be achieved easily.

The pre-specimen deflection technique can be visualized as an "inverted Bragg reflection." Usually, the incident electron beam is on optical axis and the lattice planes producing diffraction are aligned with respect to the incident beam such that they give rise to a reflection under Bragg's law. Then the reflected beam can be found at twice the Bragg angle. This picture can be inverted, such that the incident beam forms with the optical axis an angle of twice the Bragg angle. Now the reflected beam is on the optical axis (Figure 4). A deflection of the incident

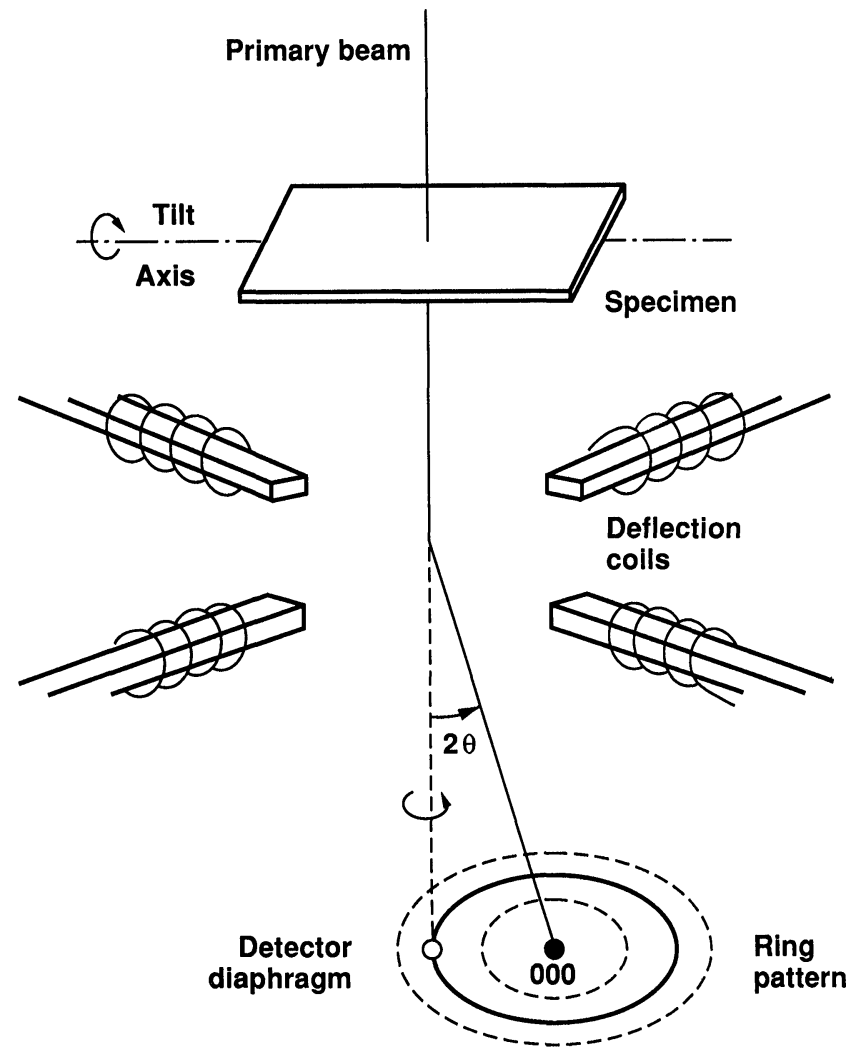

Figure 3 Post-specimen deflection. 


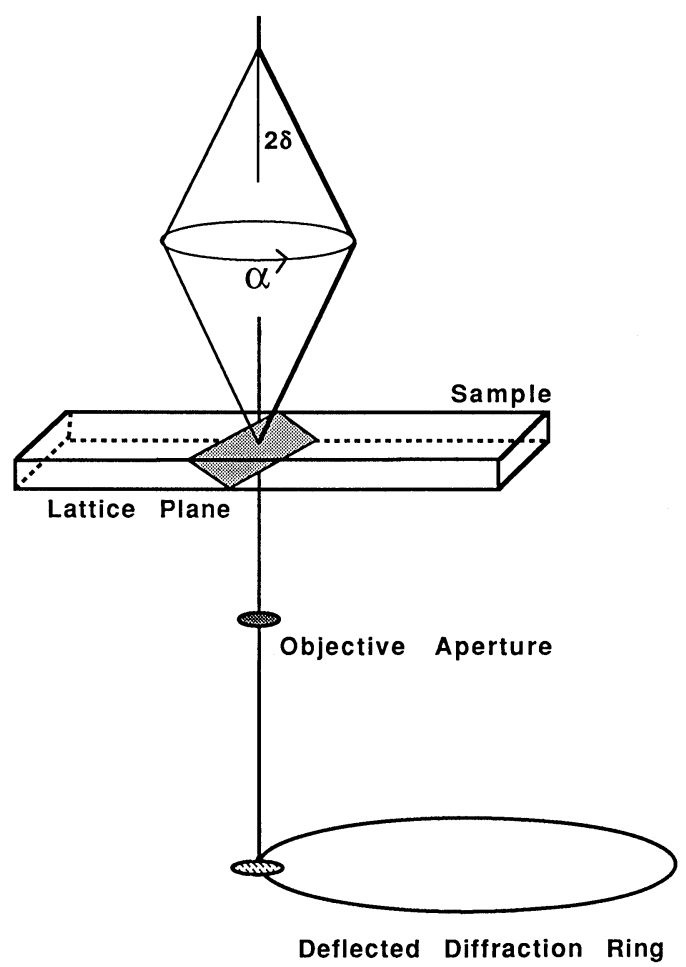

Figure 4 Pre-specimen deflection.

beam along a hollow cone with an opening angle of twice the Bragg angle then brings the deflected intensities of the according lattice planes successively onto the optical axis, where they easily can be measured with a Faraday cup. Alternatively, the intensities can be measured by the current from the fluorescent screen in the darkfield mode after inserting a small objective aperture. The later procedure allows one to observe the measured area during the analysis and to correct for any specimen drift.

\section{Set-up and Examples}

For an accurate and efficient measurement of pole figures in a TEM, control of all necessary functions by a computer is requisite. The azimuth deflection $\alpha$ (Figure 2) on each diffraction ring in both techniques requires the control of deflection coils in the microscope. The computer drives either the post-specimen deflection coils or the hollow-cone forming pre-specimen deflection coils (Figure 5). After each increment of $\alpha$ by $\Delta \alpha$, the diffracted intensity is measured and stored. Additionally, for each diffraction ring, the background is measured on both sides of a diffraction ring. The background is obtained for that value of the azimuth $\alpha$, where the diffraction ring itself showed a minimum. Thus, the influence of dispersed intensities is minimized. 


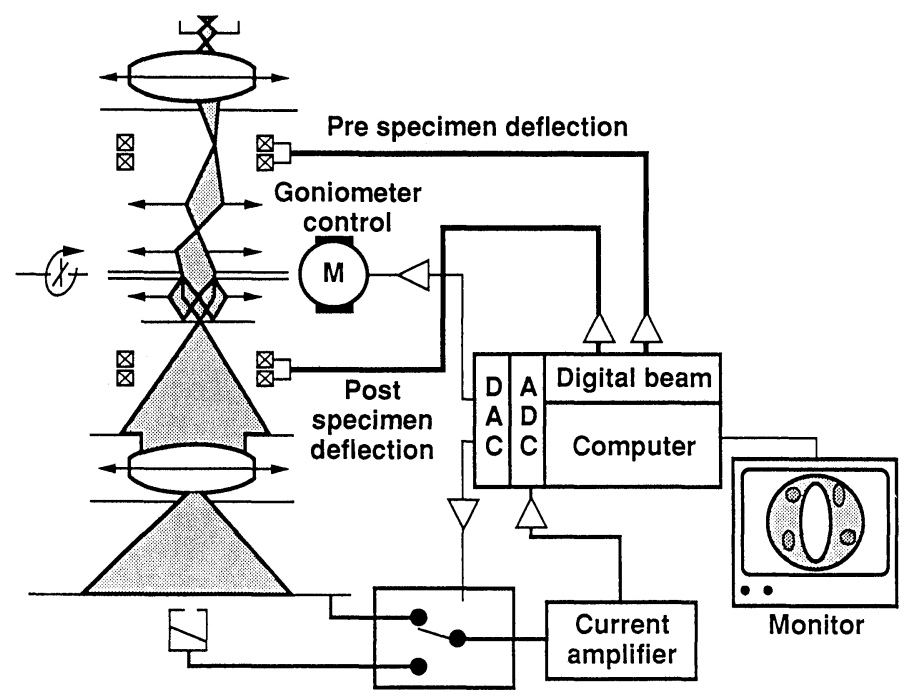

Figure 5 Set-up for on-line measurement of pole figures with a TEM.

As it will be shown later, at least three different pole figures are necessary for an ODF analysis. Therefore, three or more diffraction rings are measured at each tilt angle $\beta$. The goniometer is also controlled by the computer and is usually advanced with increments of $\beta$ in the size of $\Delta \alpha(\Delta \alpha=\Delta \beta)$.

Figure 6 shows as an example four different pole figures obtained from the same area in the matrix of an aluminum alloy deformed to a strain of $\varepsilon=2$. The lenticular shaped unmeasured area, bound by two great circles, is clearly visible. The intensities were measured for tilt angles of $-55^{\circ}$ to $+55^{\circ}$ in $5^{\circ}$ increments for $\beta$ as well as for $\alpha$. With reference to Figure 2, the tilt angle $\beta$ corresponds to the great circle in the pole figure. The maximum tilt angle possible thus defines the region of measurement. In Figure 6 , the maximum tilt angle $\beta=55^{\circ}$ is at the central location of the great circle. The pole densities in Figure 6 are corrected for background, as well as for the electron-sample interactions as described later. It is not practical to measure for each sample a reference sample with a random texture. Thus, the shown intensities are normalized with respect to the average pole density of each pole figure after the intensity correction. Therefore, the intensities are arbitrary but self-consistent.

The pole figures shown are measured in the longitudinal section of the rolled aluminum sheet. The rolling direction is aligned with respect to the tilt axis of the goniometer. Thus the transverse direction of the sample is normal to the specimen and, therefore, the transverse direction is the normal in the stereographic projection of these pole figures.

\section{QUANTITIVE ANALYSIS}

\section{Intensity Correction}

For a quantitative analysis, the measured intensities have to be corrected for the background intensity, for absorption of electrons in the specimen, and for the 


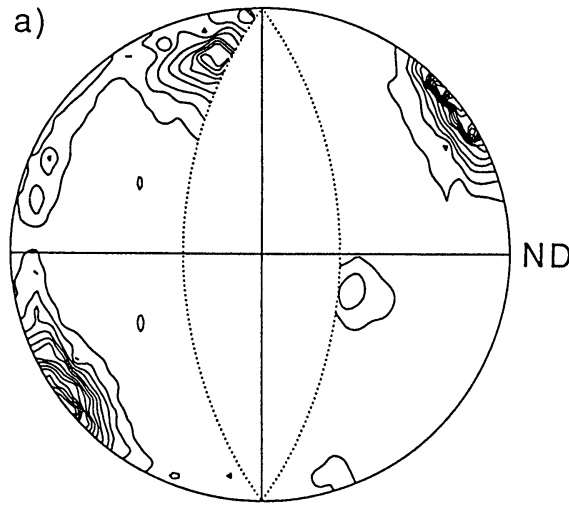

RD

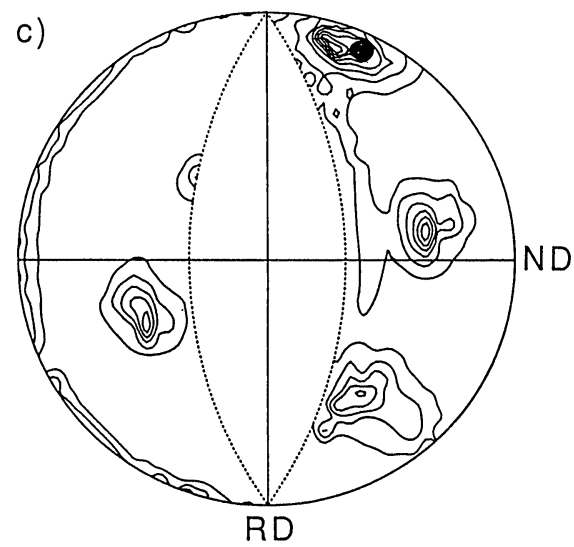

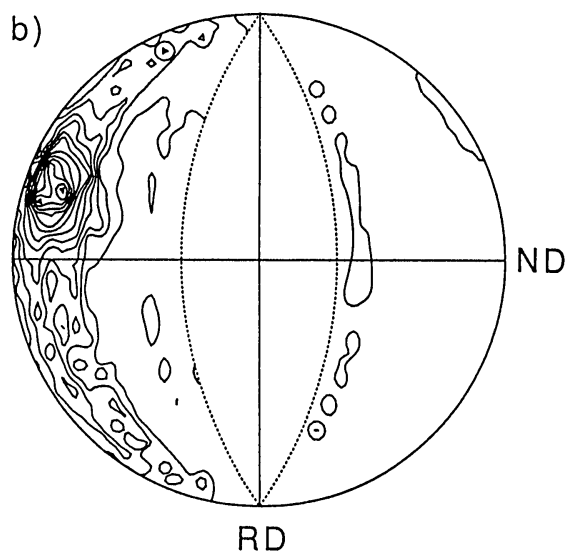

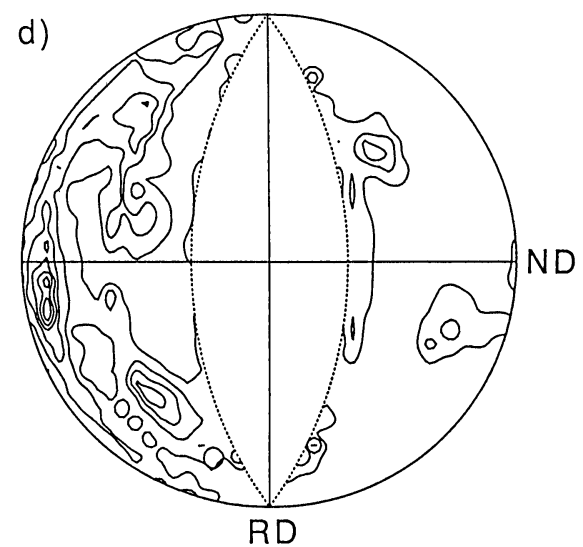

Figure 6 Pole figures from a deformed matrix in aluminum, analyzed area is $18 \mu \mathrm{m}$ in diameter. Data are from the longitudinal section of the specimen (TD normal to the plot). a) $\{111\}, b)\{002\}$, c) $\{022\}$, and d) $\{113\}$.

increase of diffracting volume with increasing tilt. In most cases the crystallite sizes are larger than the sample thickness and thus it is not necessary to take multiple diffraction into account.

The purpose of local pole figure measurement is to analyze textures on a very small scale, and the analyzed area should be kept constant during the measurement. While the risk of a potential specimen drift during the measurement is minimal in a well stabilized microscope, the diffracting volume will increase with increasing specimen tilt. This not only gives a rise in intensity, but also it has to be kept in mind, that the local texture may be different for the larger volume at high specimen tilts compared to the local texture at low tilt angles. In certain cases, e.g. for the analysis of shearbands, the specimen can be aligned such that this increase in volume takes place within the area of interest. 
The measured intensities $I_{\text {meas }}(\alpha, \beta)$ have to be corrected as follows:

$$
I(\alpha, \beta)=\left[I_{\text {meas }}(\alpha, \beta)-I_{\text {back }}(\beta)\right] \cdot K(\beta)
$$

The value of the background at the position of the diffraction ring, $I_{\text {back }}(\beta)$, is interpolated from the background measurements at both sides of the ring. $K(\beta)$ is a correction factor taking into account the absorption and the change in volume as a function of the specimen tilt. $K(\beta)$ can be determined either analytically (Schwarzer 1989) or experimentally.

\section{Analytical Intensity Correction}

The complex relationship between diffracted intensity and the individual electron diffraction conditions makes it difficult to derive a complete analytical expression for the correction of the measured pole intensities. A first approximation for $K(\beta)$ was given by Schwarzer (1989):

$$
K(\beta)=\frac{e^{-a} \cdot \cos \beta}{e^{-a / \cos \beta}}
$$

$K(\beta)$ takes into account the increase of the analyzed specimen volume by a factor of $1 / \cos \beta$, providing the diameter of the illuminating beam is constant. The parameter $a$ corrects for the absorption and has to be determined experimentally for the selected material, specimen thickness and electron beam energy (Table 1). The specimens for the measurement of $a$ were produced by vacuum evaporation. Special care was taken to avoid the formation of preferred orientations. Figure 7 shows the experimentally determined decrease of the pole intensity with increasing specimen tilt as well as the analytical curve fitted to the data according to $\mathrm{Eq}$. (2).

\section{Experimental Intensity Correction}

The value of the necessary correction of the measured pole intensities can be obtained experimentally (Schwarzer 1989). As described earlier, the SAD diffraction rings form in effect great circles in the stereographic projection. The specimen is tilted around a fixed axis. Therefore, all the rings obtained at various tilts $\beta$ intersect at the same point of the projection (Figure 2). Thus, the same pole figure point is measured for different tilt angles. The change in intensity of this point with different tilts now reflects those effects which do not originate from

Table 1 Experimentally determined values of $a$ for different electron energies and selected elements

\begin{tabular}{llll}
\hline & $A l$ & $C u$ & $A u$ \\
\hline $120 \mathrm{kV}$ & 5.4 & 4.7 & 1.83 \\
$300 \mathrm{kV}$ & 1.26 & 2.5 & \\
\hline
\end{tabular}

Specimen thickness at $120 \mathrm{kV}$ is $300 \mathrm{~nm}$, at $300 \mathrm{kV}$ $500 \mathrm{~nm}$. Data at $300 \mathrm{kV}$ from Schwarzer (1989). 


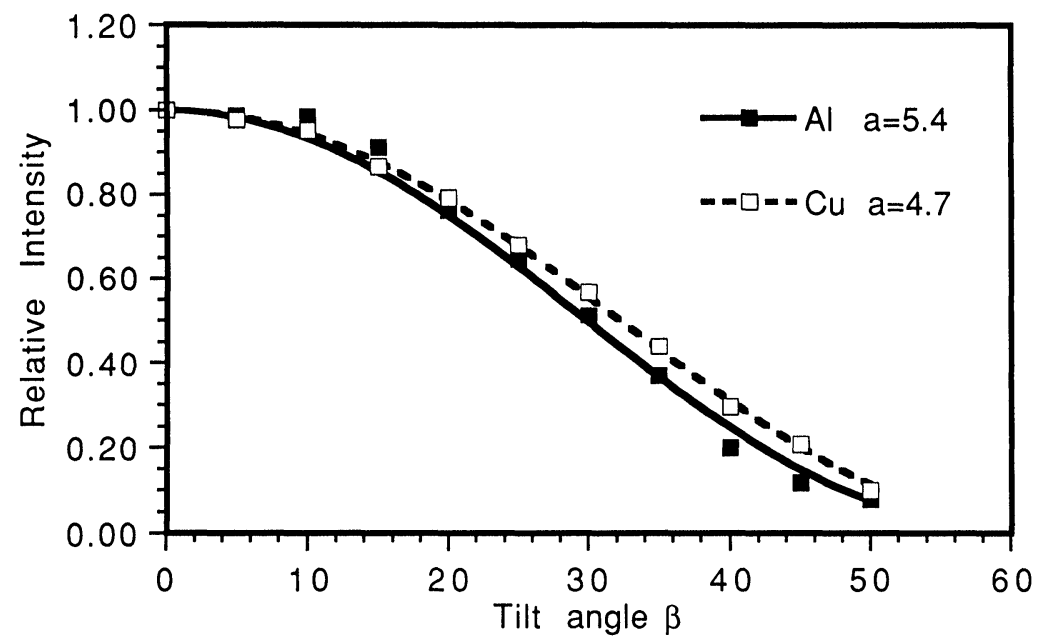

Figure 7 Decrease in diffracted intensity for randomly textured samples as a function of the specimen tilt angle $\beta$. Experimentally (squares) and analytically (solid line) determined.

texture. Therefore, for an experimentally based correction, the measured pole intensities $I_{\text {meas }}(\alpha, \beta)$ are normalized with respect to the change of intensity at this point on the pole figure.

\section{ODF Analysis}

Several methods are used for determination of orientation distribution functions (ODF) from pole figures. The most commonly used method involves series expansion using spherical harmonics (Bunge, 1982 and Roe, 1966). Here, the measured intensity distribution of a pole figure is expanded in spherical harmonics and the ODF is expanded in generalized spherical harmonics. The orientation of a crystal with respect to the sample coordinate system will be expressed through the Euler angles $\psi, \theta$ and $\phi$.

\section{Mathematical Details}

The pole normal intensity distribution $q^{j}(\zeta, \alpha)$ for the $j$ th pole figure can be expanded in spherical harmonics as follows

$$
q^{j}(\zeta, \alpha)=\sum_{l=0}^{\infty} \sum_{m=-1}^{1} Q_{l m}^{j} P_{m}^{l}(\zeta) \exp (-i m \alpha)
$$

where the superscript " $j$ " refers to the particular pole figure. $\zeta$ is related to the tilt angle $\beta$ by the following relation,

$$
\zeta=\cos \left(90^{\circ}-\beta\right)=\sin (\beta)
$$


The coefficients of the expansion are given by

$$
\begin{aligned}
Q_{l m}^{j} & =\frac{1}{2 \pi} \int_{S} q^{j}(\zeta, \alpha) P_{m}^{l}(\zeta) \exp (i m \alpha) d \zeta d \alpha \\
& =\frac{1}{2 \pi} \int_{0}^{2 \pi} \int_{-1}^{1} q^{j}(\zeta, \alpha) P_{m}^{l}(\zeta) \exp (i m \alpha) d \zeta d \alpha
\end{aligned}
$$

where $S$ refers to the integration over the entire pole sphere. The measured intensities $I_{h k l}(\xi, \alpha)$ and the pole normal intensity distribution $q^{j}(\zeta, \alpha)$ are related by

$$
q^{j}(\zeta, \alpha)=N_{h k l}^{j} I_{h k l}^{j}(\zeta, \alpha)
$$

where $N_{h k l}$ is the normalization factor for the $(h k l)$ pole figure. In the case of incomplete pole figures, Eq. (5) is rewritten in the form

$$
\begin{aligned}
Q_{l m}^{j}= & \frac{1}{2 \pi}\left[\int_{B} q^{j}(\zeta, \alpha) P_{m}^{l}(\zeta) \exp (\operatorname{im} \alpha) d \zeta d \alpha\right. \\
& \left.+\int_{S-B} q^{j}(\zeta, \alpha) P_{m}^{l}(\zeta) \exp (i m \alpha) d \zeta d \alpha\right]
\end{aligned}
$$

where $B$ refers to the region of pole sphere where pole intensities are measured and $S-B$ is the unknown region.

The orientation distribution function $w(\psi, \xi, \phi)$ can be written in terms of the generalized spherical harmonics as a function of the Euler angles $\psi, \theta$ and $\phi$. It is given by Roe (1966)

$$
w(\psi, \xi, \phi)=\sum_{l=0}^{\infty} \sum_{m=-1}^{1} \sum_{n=-1}^{1} W_{l m n} Z_{l m n}(\xi) \exp (-i m \psi) \exp (-i n \phi)
$$

$W_{l m n}$ are the coefficients of expansion, and $Z_{l m n}$ is the generalization of the associated Legendre function, with $\xi=\cos (\theta)$.

The coefficients $W_{l m n}$ are given by

$$
W_{l m n}=\left(\frac{1}{2 \pi}\right)^{2} \int_{\psi=0}^{2 \pi} \int_{\phi=0}^{2 \pi} \int_{\xi=-1}^{1} w(\psi, \xi, \phi) Z_{l m n}(\xi) \exp (\operatorname{im} \psi) \exp (\operatorname{in} \phi) d \xi d \phi d \psi
$$

The coefficients of the pole figure expansion $Q_{l m}^{j}$ are related to the coefficients $W_{l m n}$ of the ODF by

$$
Q_{l m}^{j}=2 p \sqrt{\frac{2}{(21+1)}} \sum_{n=-1}^{1} W_{l m n} P_{l}^{m}\left(\Xi^{j}\right) \exp \left(\operatorname{in} \Phi^{j}\right)
$$

with $\Xi^{j}=\cos \left(\Theta^{j}\right) . \Theta^{j}$ and $\Phi^{j}$ are the spherical coordinate system of the reciprocal vector of the $j$ th pole figure with respect to the crystal coordinate system.

In the case of complete pole figure measurements the coefficients can be directly calculated using Eq. (10). Details of the calculations and the effects of sample symmetry can be found in the literature (Bunge, 1982 or Roe, 1966 or Panchanadeeswaran, 1991). However in the case of incomplete pole figures only 
partial information is available and only the first term of Eq. (7) can be evaluated. Hence, the general expansion in Eq. (3) for pole figures is not possible. Thus the coefficients are determined by minimizing the "objective" function defined by

$$
\Delta=\sum_{j} \int_{B}\left[N^{j} I_{h k l}^{j}-q^{j}\right]^{2} d \zeta d \alpha=\text { minimum }
$$

by least squares minimization procedure. In Eq. (11), $N^{j}$ (the normalization factor) and $q^{j}$ are treated as unknowns. The index $j$ is the number of pole figures and $I_{h k l}^{j}$ are the measured pole intensities.

Equations, (3), (11) and (10) provide a set of equations for the unknown $W$ coefficients. The details of obtaining the $W$ coefficients from incomplete pole figures using the minimization procedure are given elsewhere (Bunge, 1982).

It should be pointed out that the least squares minimization procedure involves setting up a large system of matrix equations. Furthermore, the unknown normalization constants requires actual inversion of the matrix. In the case of partial reflection pole figures obtained by X-ray diffraction procedure, the area separating measured and unmeasured regions on the pole sphere depends only on the angle $\beta$. This splits up the large system of matrix equations into several small systems. This advantage is not available in the pole figures obtained by TEM methods since the region of measurement is enveloped by two great circles which depends on both angles $\alpha$ and $\beta$. This makes the positivity method more attractive.

The positivity method is an iterative procedure, developed by Dahms and Bunge (1988), and uses the condition that pole intensities must be positive. It involves artificially assuming pole intensity values in the unmeasured regions (second term in Eq. (7)) and thus generating a complete pole figure. The orientation distribution function is now determined for complete pole figures, as outlined above (Eq. (9)). From the calculated W coefficients pole figures are recalculated by Eqs. (3) and (10), and a second approximation to the pole figure in the unmeasured region can be obtained. Negative values of pole intensities, as they result from the spherical harmonics, are made equal to zero.

This iterative procedure can be continued until an appropriate convergence criterion is satisfied. Usually a good approximation to ODF and recalculated pole figures can be obtained in about 4 or 5 iterations. From the ODF obtained from the first iteration onwards additional pole figures that were not measured can be generated and used for subsequent iteration. This makes the convergence faster.

From the pole figures only the reduced ODF, for even values of the index $l$, can be obtained (Bunge, 1982 and Roe, 1966). The complete ODF is obtained by applying the same principles of the positivity method, as used in the analysis of incomplete pole figures, to the odd part of the ODF (Dahmes and Bunge, 1988).

\section{Application to Experimental Pole Figures}

Since TEM pole figures are measured in a localized region of a sample, the orthorhombic sample symmetry usually assumed in bulk texture measurements is not valid. As a result a total of 452 expansion coefficients have to be determined in place of only 124 coefficients in case of cubic-orthorhombic symmetry. 
The total number of pole figures and the region in which measurements are made are very crucial to the accuracy of orientation distributions. These have been explored in detail in a separate paper (Panchanadeeswaran and Hirsch, 1991) for a model texture. Here we will discuss the influence of experimental boundary conditions on the calculation of ODFs. In particular, we will show the influence by the number of pole figures used for ODF calculation, as well as the influence of the cut off angle $\beta_{\min }$ of the TEM goniometer on the results.

$\{111\},\{002\},\{022\}$ and $\{113\}$ pole figures (Figures $6 a$ to $6 \mathrm{~d}$ ) were measured in the matrix of a particle-containing aluminum alloy cold rolled to a strain of 2.0. The size of the measured area is about $20 \mu \mathrm{m}$. Additionally, pole figures were obtained in the deformation zone around an adjacent particle about $2.5 \mu \mathrm{m}$ in diameter. The maximum tilt angle $\beta$ is $55^{\circ}$. The TEM foils were cut along aplane containing the rolling direction and short transverse direction.
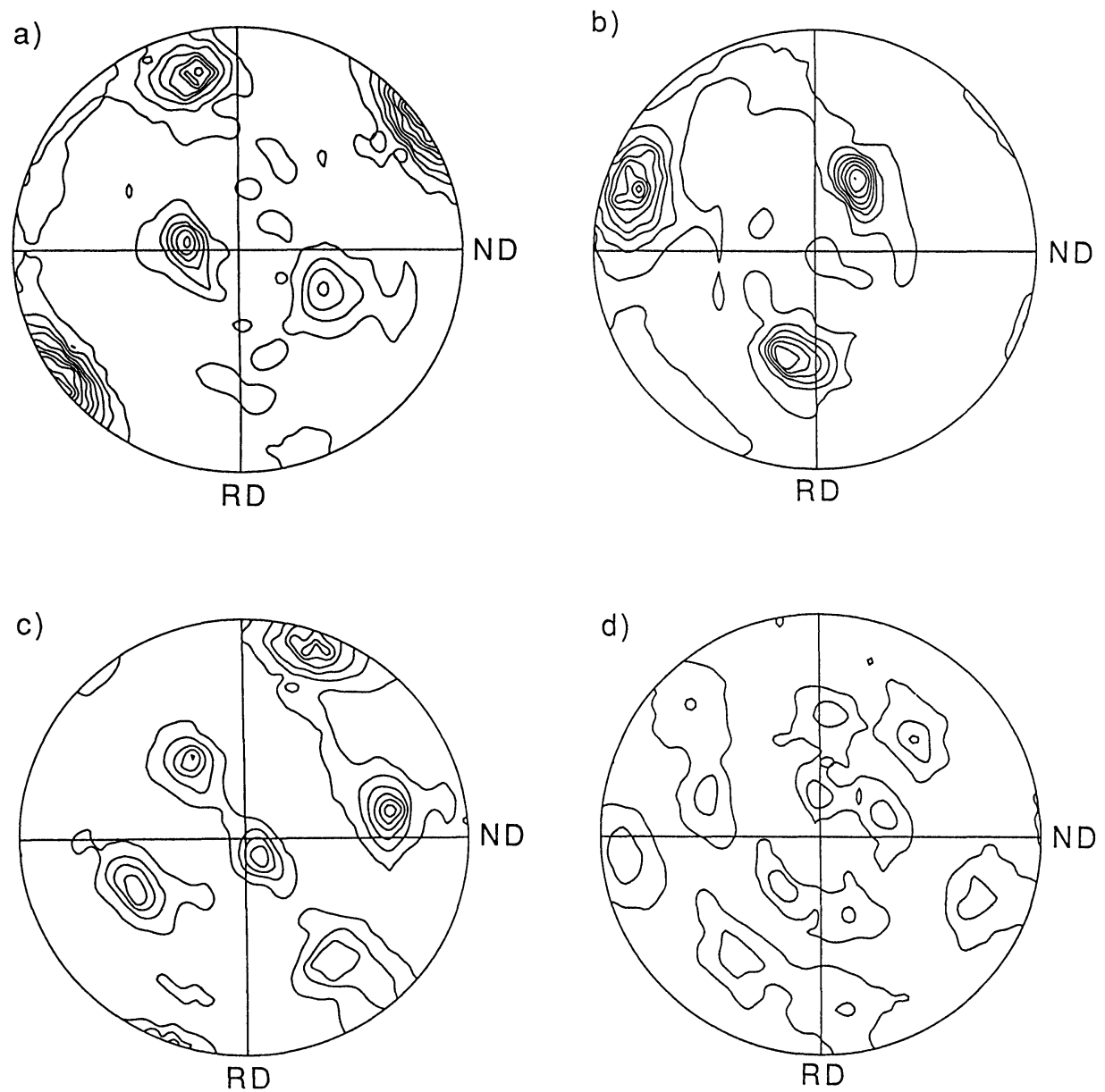

Figure 8 Recalculated pole figures to compare with Figure 6. a) $\{111\}$, b) $\{002\}$, c) $\{022\}$, and d) $\{113\}$. 
Figures $8 \mathrm{a}$ through $8 \mathrm{~d}$ show recalculated pole figures, corresponding to the experimental pole figures in Figure 6. For convenience, Figure 9 shows the same $\{111\}$ and $\{002\}$ pole figure of Figure 8 in standard pole figure convention by a $90^{\circ}$ rotation about the rolling direction. The recalculated pole figures are in good agreement with the experimental pole figures including the $\{113\}$ pole figure which was not used in the calculation of the $W$-coefficients. The experimental pole figures are not normalized with respect to a random distribution, therefore intensity levels in the recalculated pole figures are different from those in the experimental pole figures.

An advantage of the application of the positivity technique for the calculation of ODF from TEM pole figures is that the intensities calculated for the unmeasured area of the pole figure are crystallographically related to the intensities in the measured area. This suppresses intensities in the pole figures from second phases. An example of this can be seen in Figure 6c. Here the experimental $\{022\}$ pole figure shows in the upper right corner a strong intensity, which is due to a particle reflection superimposed onto the $\{022\}$ diffraction ring. This peak is missing in the reconstruction of the complete pole figure (Figure 8c), as no intensities can be found in other areas of the measured part of the pole figure, which would correspond to cubic symmetry.

The orientation distribution function was calculated for this geometry and was transformed by $90^{\circ}$ rotation around the rolling direction. This is necessary to represent ODF of rolled materials in the geometry usually used in X-ray diffraction, where ND is the normal in the stereographic projection. Figure 10 shows constant $\phi$ cross sections in $15^{\circ}$ steps of the even part of the ODF (Figure $10 \mathrm{a})$ and the complete (Figure 10b) orientation distribution function calculated from three experimental pole figures (Figure 6a-c). The increase of the maximum orientation density from the reduced (even) ODF (Figure 10a) as obtained by the pole figure inversion to the complete ODF (Figure 10b) by about $30 \%$ demonstrates the need for the calculation of the odd part of the ODF, as mentioned above. The ODF obtained shows an orientation distribution spread in

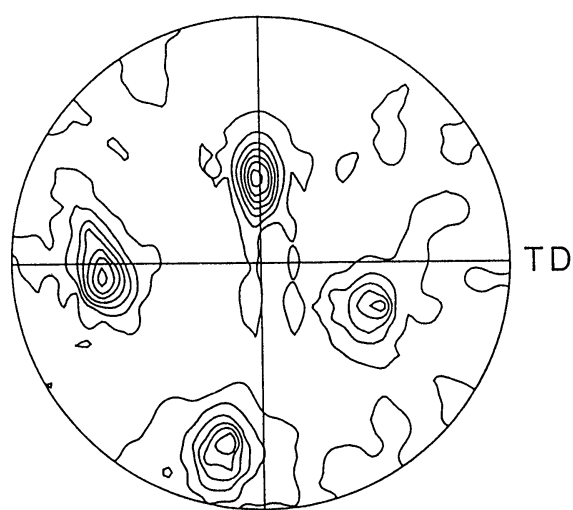

RD

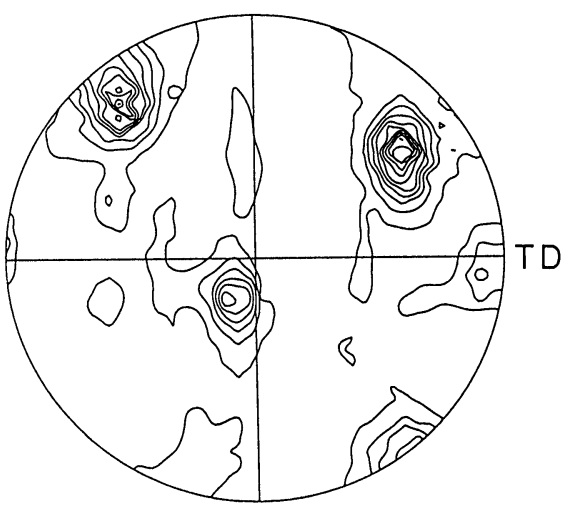

$\mathrm{RD}$

Figure 9 Recalculated pole figures as Figure 8, but in standard pole figure convention (ND normal to the plot), a) $\{111\}$, b) $\{002\}$. 


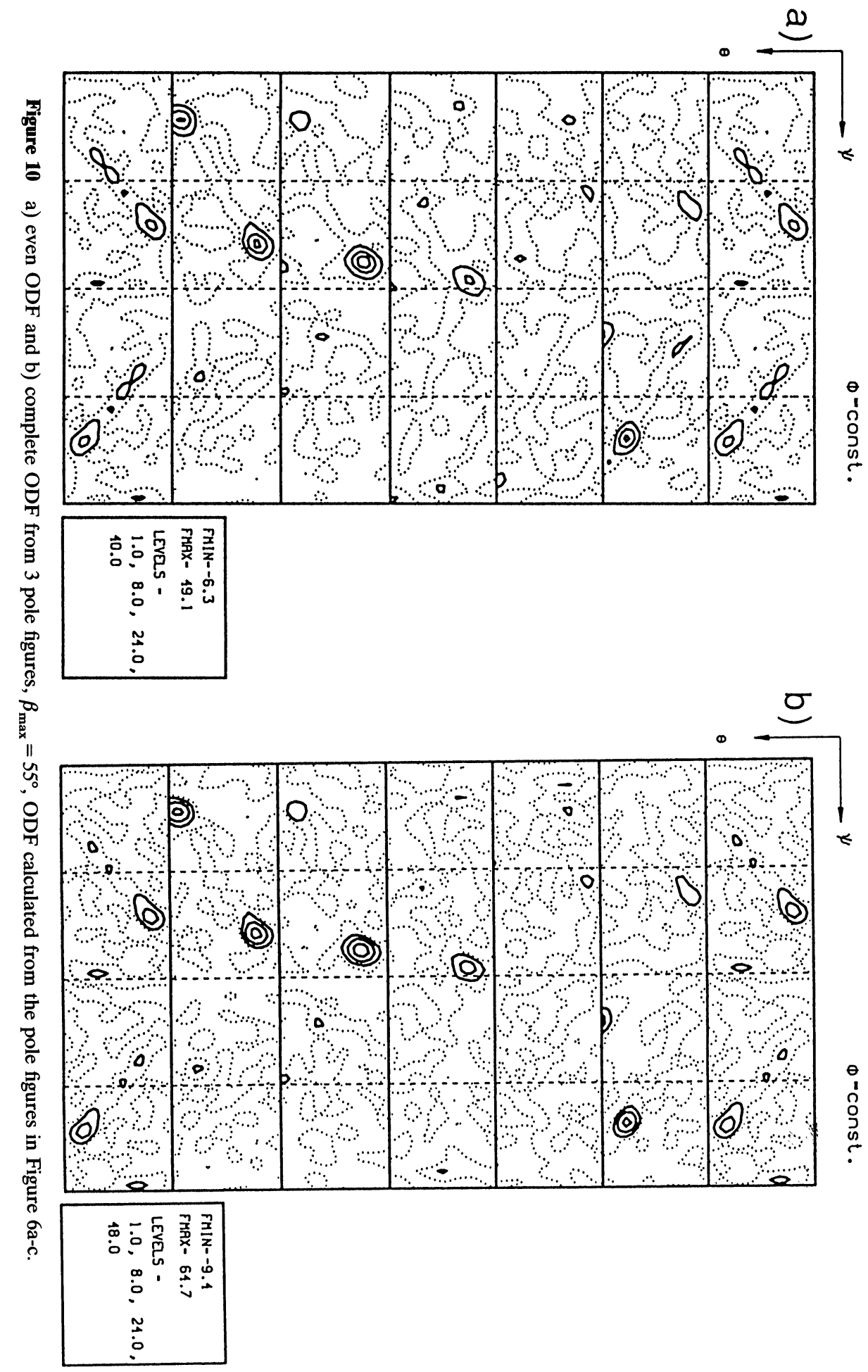




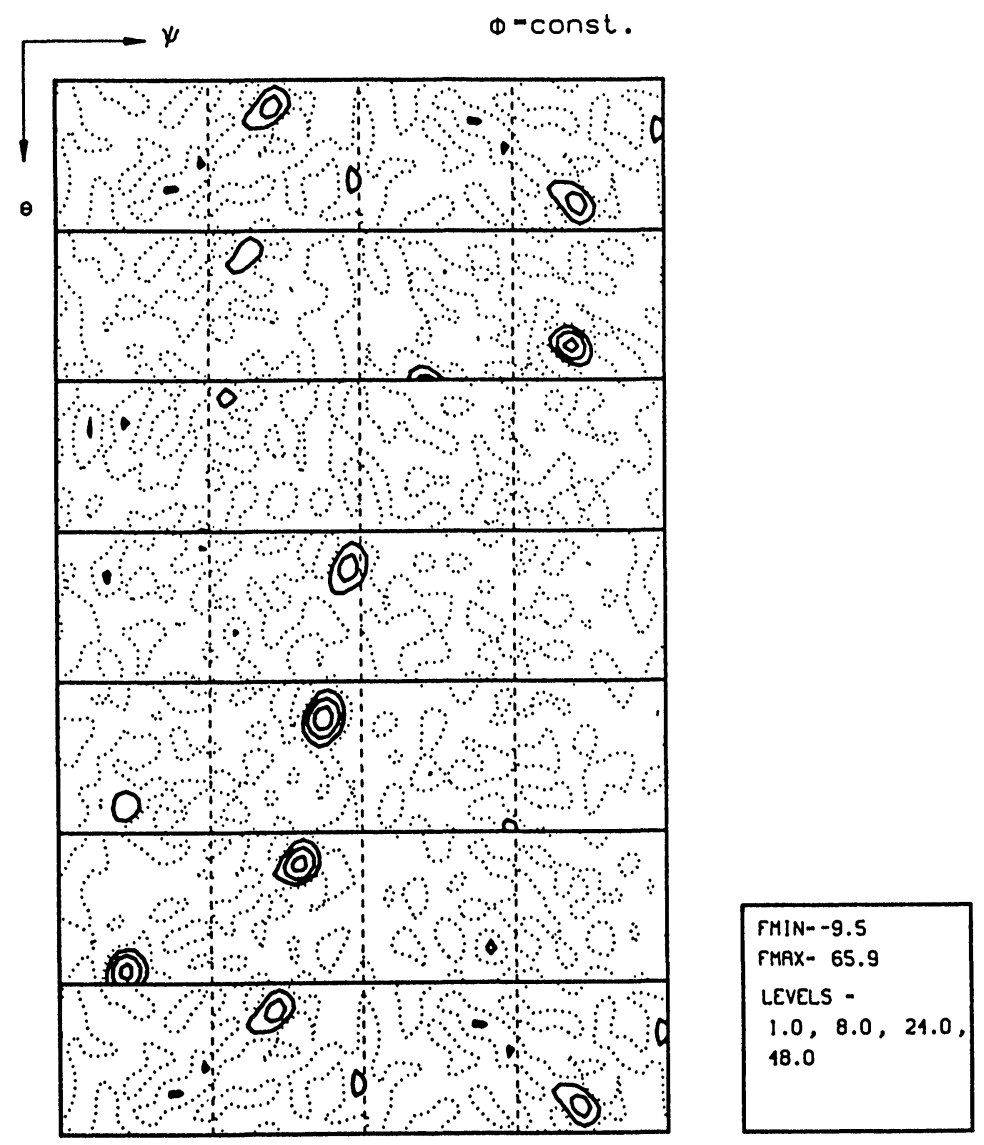

Figure 11 Complete ODF from 4 pole figures, $\beta_{\max }=55^{\circ}$.

between the $\mathrm{Cu}\left(\phi=45^{\circ}\right)$ and $\mathrm{S}\left(\phi=60^{\circ}\right)$ component of the deformation texture of face-centered cubic materials.

Increasing the input data for ODF calculation by including the $\{113\}$ pole figure and thus using four pole figures for the ODF determination (Figure 11), does not change the results significantly. Only the maximum orientation density increases slightly from $f(g)=64.7$ for the ODF from three pole figures to $f(g)=65.9$ for the ODF from four pole figures.

Reducing the information for ODF calculation by lowering the maximum tilt angle $\beta_{\max }$ from $55^{\circ}$ to $50^{\circ}$ also does not change the ODF significantly (Figures 12 and 13). The overall distribution of the orientations remains the same: the maxima of the ODF are always at the same positions and no new components appear or old components disappear. Only the maximum orientation density decreases by less than $10 \%$. A further reduction of the maximum tilt angle $\beta_{\max }$ to $45^{\circ}$ made an ODF calculation worse.

Thus it can be concluded that in this example of a very sharp texture, the measurement of three pole figures is enough for ODF calculations. Also slight 

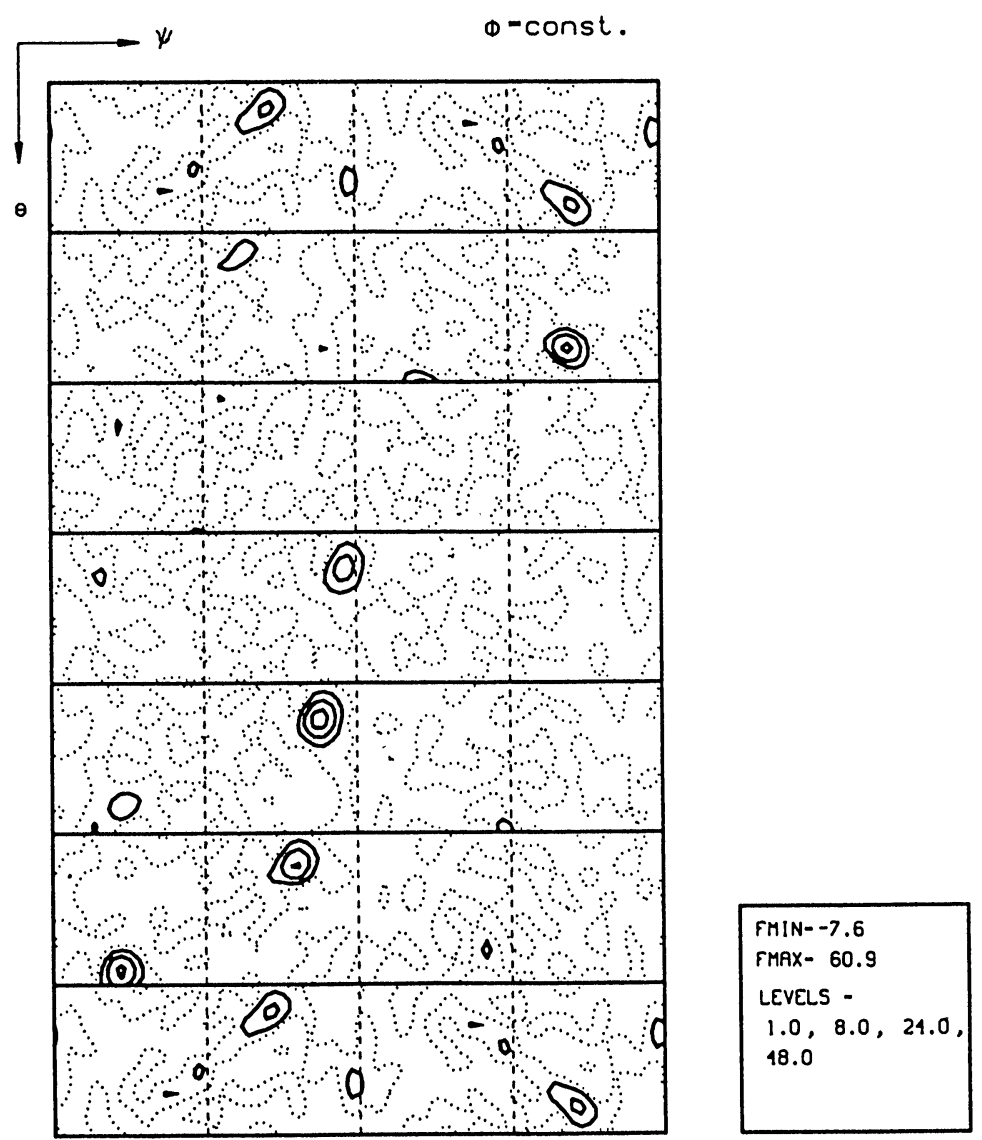

Figure 12 Complete ODF from 3 pole figures, $\beta_{\max }=50^{\circ}$.

limitations in the tilt range of the goniometer do not influence the results significantly. But it must be pointed out, that this is not necessarily valid for weak textures.

Application of least squares method to this set of pole figures did not produce any useful results (not shown). The least squares method overemphasizes minor details of the experimental pole figure, as e.g. experimental noise. This point will be clarified in the next section.

\section{TRUNCATION ERROR}

A truncation error is a result of the termination of pole figure expansion at a finite value $L$ of the index $l$. This truncation is necessary because of the limited number of experimental pole figures available. The variation of the truncation error with the rank of $l$ gives an idea of the accuracy of the experimental pole figures and the relative applicability of the different techniques to obtain ODFs 


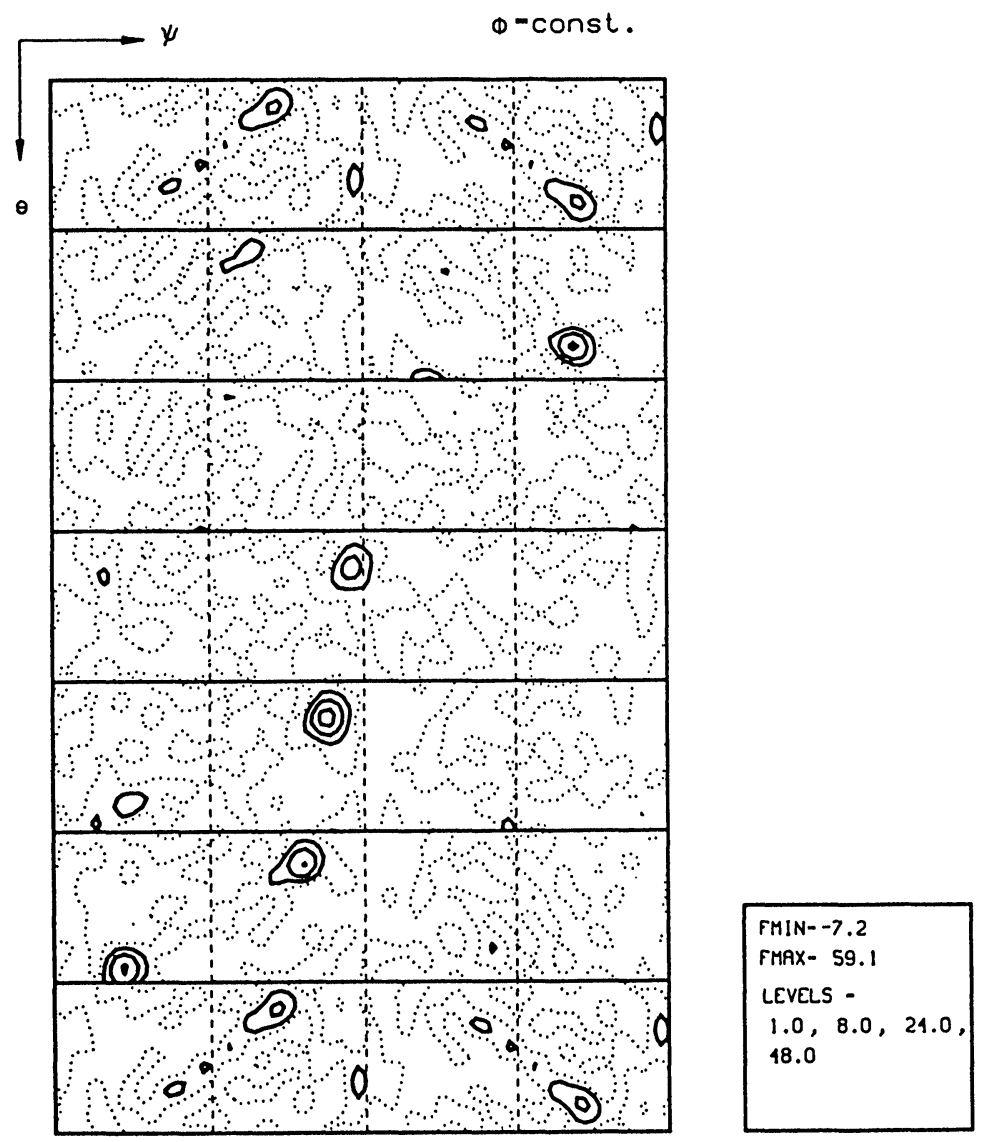

Figure 13 Complete ODF from 4 pole figures, $\beta_{\max }=50^{\circ}$.

from pole figures. The truncation error can be defined by Bunge (1982) as

$$
A_{L}=\sum_{l=L+1}^{\infty} \sum_{m=-1}^{1} \sum_{n=-1}^{1} W_{l m n}^{2}
$$

In the calculation of ODFs from pole figures the function $w(\psi, \xi, \phi)$ is not known in principle and hence the summation in Eq. (12) can not be calculated. However, the summation given by

$$
S_{L}=\sum_{m=-L}^{L} \sum_{n=-L}^{L} W_{L m n}^{2}
$$

can be plotted aginst $l$ to get an idea of the influence of pole figure measurement errors on the series expansion coefficients.

Figure 14 shows $S_{L}$ versus the rank $l$ for different techniques for the ODF calculation from experimental pole figures. No major differences in $S_{L}$ were visible for the differences in the tilt angle or for the number of pole figures used for the calculations (not shown). In order to see the influence of accuracy of pole 


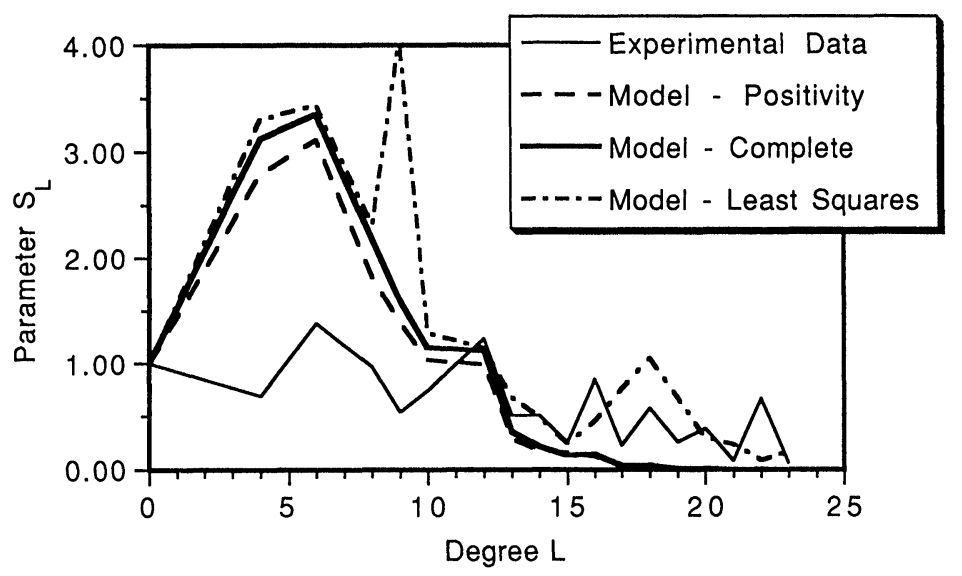

Figure $14 W$-coefficients as a function of the rank 1 .

figures on ODF a model texture in form of a Gaussian shaped peak around the major texture component in the experimental texture (Figure 10b) was generated. The coefficients $W_{l m n}$ as represented by the parameter $S_{L}$ converge for the model texture calculated from complete pole figures with increasing rank $l$ to zero (Figure 14: Model Complete). A simulation of a TEM pole figure is achieved by removing the central lens shaped area in the model pole figures. The application of the positivity method to these data shows also a fast convergence of $S_{L}$, while the least squares method does not result in a clear convergence of $S_{L}$ and the errors introduced by this method are clearly seen. The experimental data show a much slower convergence than the model texture, indicating that the experimental noise influences the calculation.

\section{LOCAL TEXTURE OF THE DEFORMATION ZONE AROUND A PARTICLE}

The texture discussed above was obtained in the matrix of a deformed aluminum alloy. Figure 15 shows the ODF of the deformation zone around a particle embedded in this matrix. The analyzed sample area is directly adjacent to the measured area of the matrix.

The maximum orientation density of the ODF in the deformation zone is decreased to half the value of the matrix. Additionally, the position of the maximum of the texture has shifted towards the ideal $\mathrm{Cu}$-component $\left(\phi=45^{\circ}\right)$. Both effects are anticipated. As the material flows during the deformation process around the particle, the crystallites rotate more strongly as the matrix is uninfluenced by the particle, thus producing a wider spread of orientations (Humphreys, 1983b). Also the deformation mode is changing in the vicinity of the particle. It may be a plane strain deformation in the matrix, but around the particle additional shear components are present. This is known to shift orientations which are, as in the matrix, only close to the $\mathrm{Cu}$-component towards the ideal Cu-orientation (Hirsch, 1990). 


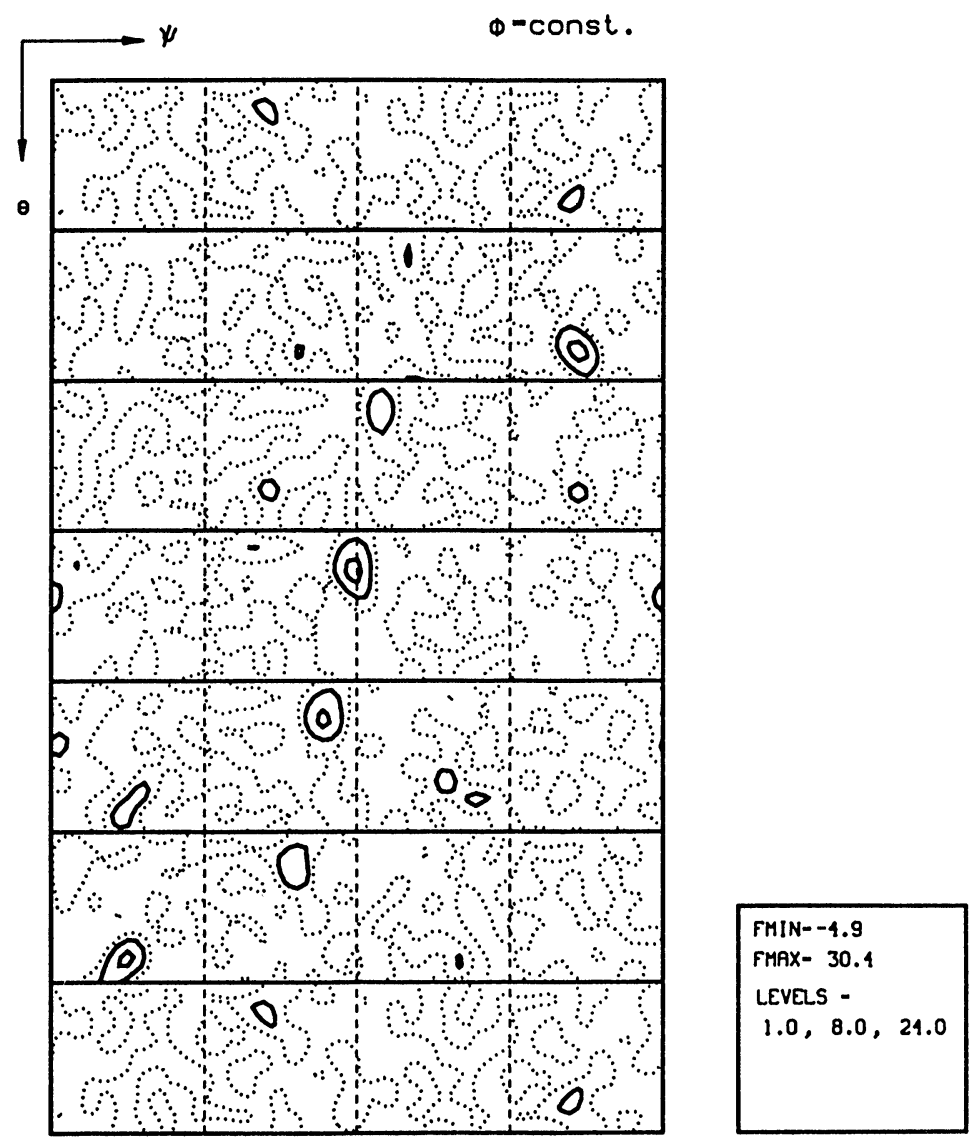

Figure 15 Texture of the deformation zone around a particle. Complete ODF calculated from 3 pole figures, $\beta_{\max }=55^{\circ}$.

\section{SUMMARY AND COMPARISON WITH OTHER MICROSCALE TECHNIQUES}

In comparison with other microscale techniques, for heavily deformed structures only the TEM based measurement of individual orientations can compete with the pole figure technique. For the applications mentioned above, the SEM based backscattered Kikuchi pattern technique lacks the necessary spatial resolution. In a local area, three incomplete pole figures are obtained within $45 \mathrm{~min}$ (Table 2), while in the same time approximately only 30 Kikuchi diagrams can be analyzed (even when a single Kikuchi diagram may be analyzed within a certain time frame, additional time is required to obtain a new pattern from the next grain). The measurement time alone does not decide the measurement technique, but more the application.

The measurement of pole figures in the TEM and the calculation of ODFs is a powerful technique to study local textures quantitatively. The main application of this technique is for analyses, where it is important to know the local texture, but 
Table 2 Measurements of crystallographic orientations by microscale techniques

\begin{tabular}{|c|c|c|c|}
\hline & \multicolumn{3}{|c|}{ Microscale techniques } \\
\hline & TEM-pole figures & $S E M-B K P$ & $T E M-K P$ \\
\hline $\begin{array}{l}\text { Penetration } \\
\text { depth (Al) }\end{array}$ & $50 \mathrm{~nm}$ & $50 \mathrm{~nm}$ & $100-200 \mathrm{~nm}$ \\
\hline $\begin{array}{l}\text { Spatial } \\
\text { resolution }\end{array}$ & $1 \mu \mathrm{m}$ & $1 \mu \mathrm{m}$ & $10 \mu \mathrm{m}$ \\
\hline Data output & Pole figures & Individual orientations & Individual orientations \\
\hline Morphology & No & Yes & Yes \\
\hline $\begin{array}{l}\text { Measurement } \\
\text { rate }\end{array}$ & $\begin{array}{l}45 \mathrm{~min} / \text { local area for } 3 \\
\text { incomplete pole figures }\end{array}$ & $\begin{array}{l}\text { 16-30 sec/subgr. } \\
\text { full/semiautomatic } \\
\text { respectively }\end{array}$ & $1 \mathrm{~min} /$ subgrain \\
\hline Specimen prep. & $3 \mathrm{~mm}$ TEM foil, critical & Bulk specimen, simple & $3 \mathrm{~mm}$ TEM foil, critical \\
\hline $\begin{array}{l}\text { Major } \\
\text { applications }\end{array}$ & $\begin{array}{l}\text { Extremely fine grained or } \\
\text { heavily deformed } \\
\text { microstructures }\end{array}$ & $\begin{array}{l}\text { Recrystallized or } \\
\text { recovered substructures }\end{array}$ & $\begin{array}{l}\text { Deformed microstructures, } \\
\text { recrystallization nuclei }\end{array}$ \\
\hline
\end{tabular}

BKP: Backscattered Kikuchi Patterns, KP: Kikuchi Patterns

where the knowledge of misorientations and of the spatial arrangement of orientations, the morphology, is unimportant. Typical applications of this technique are the texture measurement of thin films, the study of deformation inhomogeneities and the determination of the texture of the parent matrix around a recrystallization nucleus. In the later case, the parent matrix is determined by the local pole figure technique, while the orientation of the nucleus is determined from a Kikuchi pattern in convergent beam technique.

\section{References}

Bunge, H. J. (1982). Texture Analysis in Materials Science, Butterworth Publ., London.

Dahms, M. and Bunge, H. J. (1988). Proceedings ICOTOMS, Eds. J. S. Kallend and G. Gottstein, Santa Fe, New Mexico, 79.

Hirsch, J. R. (1990). Proc. Recrystallization '90, Wollongong, Australia, ed. T. Chandra, TMS publ., 759.

Humphreys, F. J. (1983a). Textures and Microstructures 6, 45-62.

Humphreys, F. J. (1983b). Proc. 4th Int. Risø Symp., ed: J. B. Bilde-Sørensen et al., p. 41-52.

Panchanadeeswaran, S. and Hirsch, J. (1991). Textures and Microstructures, 14-18 225-230.

Roe, R. J. (1966). Journal Appl. Phys., 36, 2024.

Schwarzer, R. A. (1982). Z. Metallkunde 73, 495-498.

Schwarzer, R. A. and Weiland, H. (1988). In: Experimental Techniques of Texture Analysis, ed. H. J. Bunge DGM Informationsgesellschaft, Oberursel, 287-300.

Schwarzer, R. A. (1989). Die Bestimmung der lokalen Textur mit derm Elektronenmikoskop, Habilitation Thesis, TU Clausthal, Germany.

Weiland, H. and Schwarzer, R. (1985). In: Experimental Techniques of Texture Analysis, ed. H. J. Bunge, DGM Informationsgesellschaft Oberursel, 301-313. 